\title{
Effects of topical dexamethasone in postoperative sore throat
}

\author{
Jeong Han Lee ${ }^{1}$, Soo Bin Kim ${ }^{1}$, Wonjin Lee ${ }^{1}$, Seunghee Ki ${ }^{1}$, \\ Myoung-Hun Kim ${ }^{1}$, Kwangrae Cho ${ }^{1}$, Se Hun Lim ${ }^{1}$, Kun Moo Lee ${ }^{1}$, \\ Deul-Nyuck Choi ${ }^{1}$, and Minkyung $\mathrm{Oh}^{2}$ \\ Departments of ${ }^{1}$ Anesthesiology and Pain Medicine, ${ }^{2}$ Pharmacology and Clinical Trial Center, Busan Paik Hospital, \\ Inje University College of Medicine, Busan, Korea
}

Background: Postoperative sore throat (POST) is a complication that undermines patient satisfaction and increases discomfort in the postoperative period. The present study examined the effects of dexamethasone gargle and endotracheal tube cuff soaking on the incidence and severity of POST.

Methods: Ninety patients undergoing laparoscopic cholecystectomy were randomly allocated into three groups: $0.9 \%$ normal saline gargling and tube soaking (group C), $0.05 \%$ dexamethasone solution gargling and $0.9 \%$ normal saline tube soaking (group G), $0.9 \%$ normal saline gargling and $0.05 \%$ dexamethasone tube soaking (group S). The incidence and severity of POST were then assessed and recorded at 24 hours after surgery.

Results: The total incidence of POST was significantly different among the groups $(\mathrm{P}<0.05)$, and group $\mathrm{S}$ exhibited a significantly lower incidence of POST than group $\mathrm{C}(\mathrm{P}<0.0167)$. In addition, the POST intensity of group $\mathrm{G}$ and group $\mathrm{S}$ was less severe than those of group C (Both $\mathrm{P}<0.0167)$.

Conclusions: Among patients undergoing laparoscopic cholecystectomy, those who gargled with $0.05 \%$ dexamethasone solution exhibited lower severity of POST than the control group, and those whose endotracheal tube cuff was soaked in the dexamethasone solution before intubation exhibited significantly lower incidence and severity of POST than the control group.

Key Words: Dexamethasone, Intratracheal intubation, Laparoscopic cholecystectomy, Pharyngitis, Postoperative complication.

Corresponding author: Jeong Han Lee, M.D., Ph.D.

Department of Anesthesiology and Pain Medicine, Busan Paik Hospital, Inje University College of Medicine, 75, Bokji-ro, Busanjingu, Busan 47392, Korea

Tel: 82-51-890-6520, Fax: 82-51-898-4216

Email: ljh646@inje.ac.kr

ORCID: http://orcid.org/0000-0002-4616-0355

Received: May 3, 2016.

Revised: June 30, 2016 (1st); August 10, 2016 (2nd); August 22, 2016 (3rd); August 30, 2016 (4th).

Accepted: September 4, 2016.

Korean J Anesthesiol 2017 February 70(1): 58-63

https://doi.org/10.4097/kjae.2017.70.1.58

\section{Introduction}

Postoperative sore throat (POST) is a common postoperative complication experienced by $10-62 \%$ of surgical patients who undergo intubation $[1,2]$. It occurs as a result of intubationinduced damage to the laryngeal and airway mucosa and extension of cervical muscles and ligaments [3]. POST induces insomnia and memory impairment, increases patient morbidity, and can delay a patient's return to normal routine activities. Furthermore, POST makes the surgery an unpleasant memory for patients following discharge [4-7].

Prior studies have found that ketamine [4] and tramadol solutions [8] for gargles, endotracheal tube spraying with beclomethasone [9], and intravenous injection of dexamethasone are effective in reducing the incidence and severity of POST [10].

(c) This is an open-access article distributed under the terms of the Creative Commons Attribution Non-Commercial License (http://creativecommons.org/ licenses/by-nc/4.0/), which permits unrestricted non-commercial use, distribution, and reproduction in any medium, provided the original work is properly cited. 
Dexamethasone is a corticosteroid that has 26.6 and 6.6 times stronger anti-inflammatory and immunosuppressant effects than cortisol and prednisone, respectively. Owing to its anti-inflammatory effects, dexamethasone is also used for autoimmune conditions such as rheumatoid arthritis and bronchospasm, for the control of side effects after chemotherapy in cancer patients [11], and as a gargle solution in dentistry to alleviate the symptoms of oral lichen planus [12].

We presumed that dexamethasone would exert its pharmacological effects in gargling and soaking through direct contact with mucosa. However, there has been no research on the effects of the topical use of dexamethasone, with techniques such as gargling and soaking endotracheal tubes, on the incidence and severity of POST.

Hence, the present study sought to examine how preoperative dexamethasone gargle and endotracheal tube soaking in dexamethasone affect the incidence and severity of POST in patients undergoing laparoscopic cholecystectomy.

\section{Materials and Methods}

This study examined patients aged between 20-70 years and with American Society of Anesthesiologists (ASA) physical status classification I or II who were undergoing laparoscopic cholecystectomy as an elective procedure. The following patients were excluded from the study: patients with recent throat pain or symptoms of upper airway infection; patients with longterm use of anti-inflammatory drugs such as non-steroidal anti-inflammatory drugs or corticosteroids; patients who had undergone the insertion of devices that stimulate the oral cavity, pharynx, or larynx, such as nasogastric tubes or endoscopic nasobiliary drainage tubes; and patients with a history of POST.

Approval for the current study was obtained from the hospital's Institutional Review Board, and written informed consent was obtained from patients after the provision of thorough explanations regarding the study. This study was a double-blind experiment in which a nurse delivered a syringe with medication that displayed only the patient's name to the anesthesiologist for the performance of endotracheal tube soaking and gargle. The anesthesiologist then performed induction, intubation, and extubation. All procedures were performed by an anesthesiologist with 15 years of experience.

The present study was performed by dividing 90 subjects into three groups. The first group was the control group (group C); these patients gargled with $0.9 \%$ normal saline for 5 seconds at a time point 10 minutes prior to the surgery, and their endotracheal tube cuff was soaked in $0.9 \%$ normal saline for 10 minutes before intubation. The second group was the gargle group (group $\mathrm{G})$; these patients gargled with dexamethasone solution (5 $\mathrm{mg}$ / normal saline $10 \mathrm{ml}, 0.05 \%$ ) for 5 seconds at a time point 10 minutes prior to the surgery, and their endotracheal tube cuff was soaked in $0.9 \%$ normal saline for 10 minutes before intubation. The third group was the soaking group (group S); these patients gargled with $0.9 \%$ normal saline for 5 seconds at a time point 10 minutes prior to the surgery, and their endotracheal tube cuff was soaked in dexamethasone solution for 10 minutes before intubation. For soaking, a $10 \mathrm{ml}$ syringe was filled with $0.05 \%$ dexamethasone solution and the tip was blocked with a piece of rubber. Then, after removing the syringe plunger, the entire cuff area of the endotracheal tube was immersed in the solution in the vertical position for 10 minutes.

The patients were premedicated 30 minutes prior to surgery with $0.2 \mathrm{mg}$ of glycopyrrolate via intramuscular injection, and upon entering the operating room, the patients were monitored with non-invasive pulse oximetry, electrocardiography, and a non-invasive blood pressure monitoring device. A tube without warming and humidifying functions was used, and anesthesia was induced with propofol $(2 \mathrm{mg} / \mathrm{kg})$ and lidocaine $(1 \mathrm{mg} / \mathrm{kg})$. Rocuronium $(0.6 \mathrm{mg} / \mathrm{kg})$ was used for neuromuscular blockade. Intubation was performed after confirming that the train of four (TOF) count (TOF-Watch SX ${ }^{\circledR}$, Organon Ltd., Dublin, Ireland) had reached 0 . End-tidal $\mathrm{CO}_{2}$ was then monitored. The patients included in the trial or this trial were all Cormack-Lehane grade I, and successful intubation was performed within two attempts. The laryngoscope used for intubation was a Macintosh \#3 (Mac Laryngoscope, Welch Allyn Inc., Skaneateles Falls, NY, USA), and a stylet was not used. A $7.5 \mathrm{~mm}$ internal diameter tube and a high-volume/low-pressure cuff (Sheridan/CF ${ }^{\circledR}$, Teleflex Medical, Morrisville, NC, USA) were used for male patients, while a $7.0 \mathrm{~mm}$ internal diameter tube was used for female patients. An oral airway (Ace Grip Endo Fix, Ace Medical Co., Ltd., Seoul, Korea) was fixed with the endotracheal tube for all patients. Anesthesia was maintained with sevoflurane 1.5-2.5 vol\%, 50\% $\mathrm{O}_{2}$ with air, and $0.05-0.1 \mu \mathrm{g} / \mathrm{kg} / \mathrm{min}$ remifentanil, while cuff pressure from the beginning to the end of the procedure was maintained at $20-30 \mathrm{cmH}_{2} \mathrm{O}$ with a non-invasive manometer (Mallinckrodt ${ }^{\mathrm{TM}}$ Hand Pressure Gauge, Covidien Deutschland $\mathrm{GmbH}$, Neustadt an der Donau, Germany). At 10 minutes prior to the completion of the procedure, $0.5-1 \mathrm{mg} / \mathrm{kg}$ of tramadol and $0.3 \mathrm{mg}$ of ramosetron were intravenously injected to prevent postoperative pain, vomiting, and nausea. Following the surgery, endotracheal suction was not performed, and only oral suction was performed. Sugammadex ( $2 \mathrm{mg} / \mathrm{kg}$ ) was injected to reverse neuromuscular blockade, and after confirming that the TOF ratio exceeded 0.9 and the patient had recovered consciousness, extubation was performed smoothly.

A different anesthesiologist (not the anesthesiologist who performed anesthesia during the procedures) visited the patients at 24 hours following extubation to assess postoperative pain. As per the classification suggested by Bagchi et al. [13], the 
study subjects were categorized into patients with no throat pain (grade, 0 ), patients who only became aware of throat pain after being asked if they had throat pain (mild throat pain, 1), patients who complained of throat pain before being asked (moderate throat pain, 2), and patients experiencing distinct discomfort due to throat pain (severe throat pain, 3). The total incidence of POST was calculated by dividing the number of patients who experienced postoperative throat pain at least once by the total number of patients. The patients were then examined to determine the occurrence of any other postoperative complications (excluding POST), and the findings were recorded in detail.

In the general wards after surgery, ketorolac $0.3-0.5 \mathrm{mg} / \mathrm{kg}$ was intravenously injected three times a day to control abdominal pain. If postoperative pain exceeded a visual analogue scale score of 5 , an additional $0.5 \mathrm{mg} / \mathrm{kg}$ of tramadol was injected in- travenously.

Patients who had a sore throat severity grade of 2 or higher and required medical intervention were administered a benzydamine gargle or an intravenous injection of ketorolac to alleviate the symptoms and were carefully observed to determine the presence of any other complications or side effects.

Before beginning the present study, we performed a power calculation based on previous study results to determine the sample size. Park et al. [14] found that among patients who received intravenous injection of prophylactic dexamethasone, the incidence of POST was $0.69 \%$. In the study of Rudra et al. [15], the incidence of POST after ketamine gargle was $25 \%$. If we assumed that the POST reduction effects generated by endotracheal tube cuff soaking would be similar to those achieved by gargling, the required number of subjects was calculated to

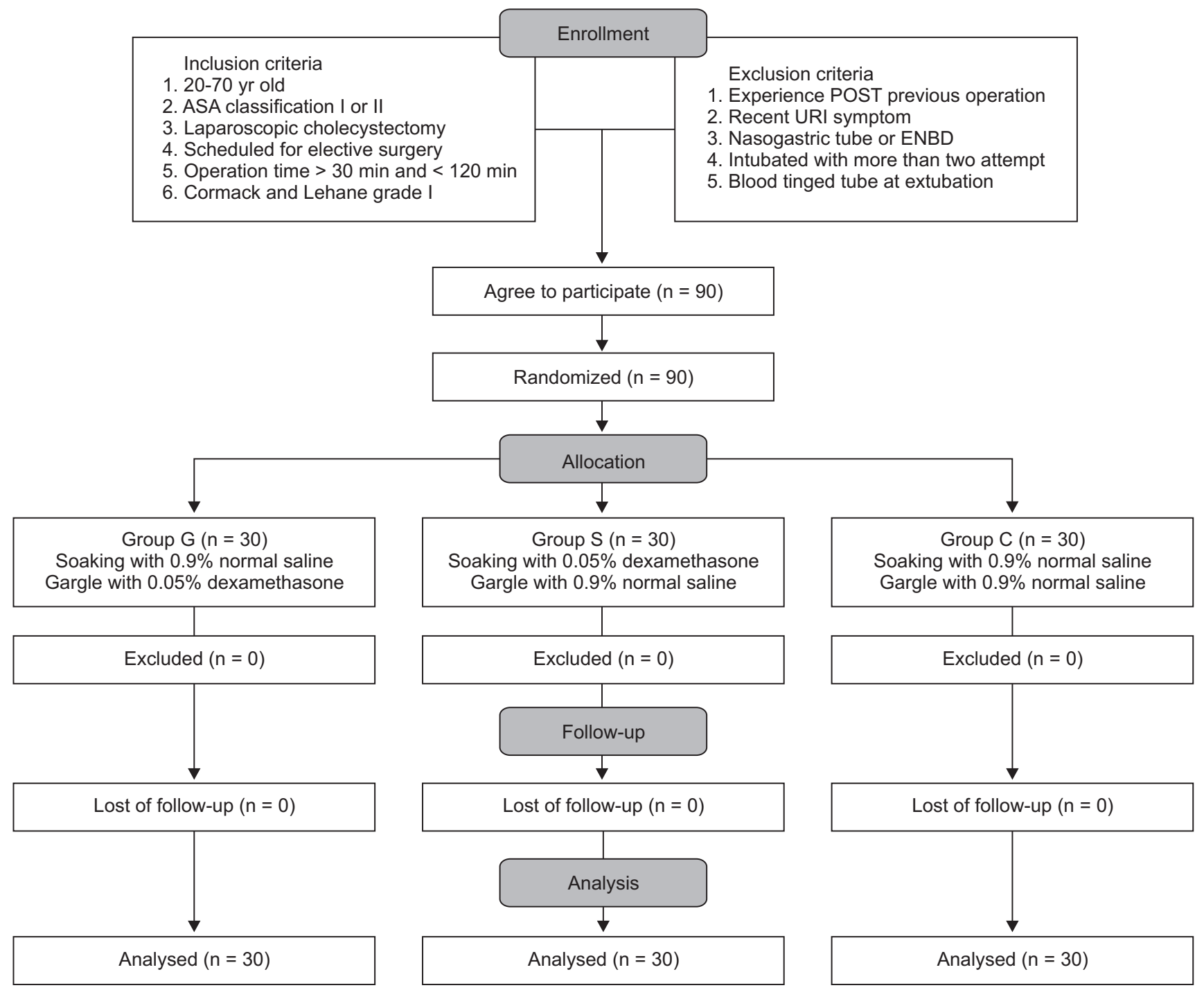

Fig. 1. Flow diagram of study enrollment. ASA: American Society of Anesthesiologists, POST: postoperative sore throat, URI: upper respiratory tract infection, ENBD: endoscopic nasobiliary drainage. 
be 26 with a significance level of $0.05 / 3=0.0167$ and a statistical power of $80 \%$. Therefore, in consideration of dropout rates, we recruited 30 subjects for each group.

Results are expressed as mean $\pm \mathrm{SD}$, median with range, or number (percentage). Patient age, height, weight, and duration of surgery were compared among groups and tested statistically using analysis of variance. The incidence of sore throat and hoarseness were tested with the $\chi^{2}$ test. To avoid a type I error, for variables found to be significant in the $\chi^{2}$ test, we recalculated all possible three $2 \times 2 \chi^{2}$ tests by applying the Bonferroni inequality to adjust the $\alpha$ level (i.e., $\mathrm{P}\left[\chi^{2}>5.73\right]=0.05 / 3=0.0167$ for 1 degree of freedom). A Kruskal-Wallis test followed by the Dunn procedure was applied to compare differences in the severity of POST among groups. Statistical analyses were conducted using SAS 9.3 (SAS Institute Inc., Cary, NC, USA). A P value $<0.05$ was considered statistically significant.

\section{Results}

Ninety patients were assessed for eligibility and no patients were excluded after screening. All patients completed the study (Fig. 1). None of the patients experienced postoperative complications.

There were no significant intergroup differences in the gender ratio, age, length of operation, ASA classification, height, weight,

Table 1. Baseline Characteristics

\begin{tabular}{lcccc}
\hline & $\begin{array}{c}\text { Group C } \\
(\mathrm{n}=30)\end{array}$ & $\begin{array}{c}\text { Group G } \\
(\mathrm{n}=30)\end{array}$ & $\begin{array}{c}\text { Group S } \\
(\mathrm{n}=30)\end{array}$ & P value \\
\hline Sex $(\mathrm{M} / \mathrm{F})$ & $12 / 18$ & $15 / 15$ & $15 / 15$ & 0.677 \\
Age $(\mathrm{yr})$ & $47 \pm 13$ & $48 \pm 12$ & $48 \pm 12$ & 0.773 \\
ASA (I/II) & $16 / 14$ & $14 / 16$ & $17 / 13$ & 0.739 \\
Height $(\mathrm{cm})$ & $157 \pm 7$ & $158 \pm 6$ & $157 \pm 6$ & 0.656 \\
Weight $(\mathrm{kg})$ & $65 \pm 9$ & $64 \pm 9$ & $62 \pm 10$ & 0.754 \\
BMI $\left(\mathrm{kg} / \mathrm{m}^{2}\right)$ & $25 \pm 2$ & $25 \pm 2$ & $25 \pm 3$ & 0.664 \\
Operation time (min) & $48 \pm 9$ & $47 \pm 15$ & $45 \pm 10$ & 0.459 \\
Hoarseness incidence & $8(27 \%)$ & $11(37 \%)$ & $4(13 \%)$ & 0.115 \\
\hline
\end{tabular}

Data presented as mean \pm SD or number of patients. Group C: control group, Group G: 0.05\% dexamethasone gargle group, Group S: 0.05\% dexamethasone endotracheal tube soaking group. ASA: American Society of Anesthesiologists, BMI: body mass index. body mass index, and in the incidence in hoarseness (Table 1).

There was a significant difference in the total incidence of POST among the three groups (Table 2$)(\mathrm{P}<0.05)$. In terms of intergroup differences, there was a significant difference in the incidence of POST between group C and group S $(\mathrm{P}<0.0167)$.

There was a significant difference among the three groups in the median values of severity of POST severity grade (Table 2) $(\mathrm{P}<0.05)$, with a significantly lower median value of severity observed in group $\mathrm{C}$ than those in group $\mathrm{C}$ (Both $\mathrm{P}<0.0167)$.

\section{Discussion}

The findings of this study indicated that gargle with $0.05 \%$ dexamethasone solution is effective in lowering the severity of POST, and endotracheal tube soaking with $0.05 \%$ dexamethasone solution is effective in lowering the incidence and severity of POST.

In a study by Saleem and Athar [16], the incidence of POST following intubation was performed using polyvinylchloride without administration of any drugs was $73 \%$. Similarly, the incidence of POST in the control group was $63 \%$ in the present study. The two most prominent causes of POST are trauma induced by tracheal intubation and necrosis of airway mucosa caused by the pressure of the endotracheal tube cuff $[4,17]$. Therefore, the incidence and severity of POST are known to increase in the following situations: when cuff pressure is increased due to the use of nitrous oxide [3]; when succinylcholine is used during obstetric and gynecologic surgery on young women [18]; when the length of the operation is too long or operative positions are changed too frequently; when too many intubation attempts are performed; when the physician performing the intubation is inexperienced [19]; or when polyvinylchloride tubes are used instead of armored tubes [16]. In prior studies, techniques such as using intubation methods with minimal irritation, performing intubation after achieving complete neuromuscular blockade, or using small endotracheal tubes [19] have been found to be effective in reducing POST. Furthermore, preoperative administration of gabapentin as a preventive measure [5], the use of benzydamine hydrochloride spray on the semipermeable cuff of the endotracheal tube [20],

Table 2. Incidence and Severity of Postoperative Sore Throat

\begin{tabular}{|c|c|c|c|c|c|c|c|}
\hline \multirow{2}{*}{ Evaluation } & \multirow{2}{*}{$\begin{array}{l}\text { Group C } \\
(\mathrm{n}=30)\end{array}$} & \multirow{2}{*}{$\begin{array}{l}\text { Group G } \\
(\mathrm{n}=30)\end{array}$} & \multirow{2}{*}{$\begin{array}{l}\text { Group S } \\
(\mathrm{n}=30)\end{array}$} & \multirow{2}{*}{$P$ value } & \multicolumn{3}{|c|}{ Odds ratio } \\
\hline & & & & & C vs. G & C vs. S & G vs. S \\
\hline Total incidence & $19(63 \%)$ & $10(33 \%)$ & $8(27 \%)^{*}$ & 0.008 & $3.45(0.54-5.43)$ & $4.75(0.60-6.48)$ & $1.38(0.34-3.83)$ \\
\hline Severity of POST & $1(0-3)$ & $0(0-1)^{*}$ & $0(0-1)^{*}$ & 0.002 & & & \\
\hline
\end{tabular}

Values are presented as number of patients or odds ratio ( $98.33 \%$ confidence interval) or median (range). POST: postoperative sore throat. Group C: control group, Group G: 0.05\% dexamethasone gargle group, Group S: 0.05\% dexamethasone endotracheal tube soaking group. *P value $<0.0167$ compared with Group C. 
and the application of betamethasone gel and lidocaine jelly on the cuff [21], as well as filling the cuff with $4 \%$ lidocaine instead of air [22], have been observed to be effective in reducing POST.

Corticosteroids such as dexamethasone generally reduce edema by hindering the emigration of leukocytes as well as the migration of plasma components, and stop the swelling of cells beyond a certain extent by increasing the stability of cellular membranes. Furthermore, these drugs inhibit hydrolysis around cells, hamper the release of lysozymes from granulocytes and phagocytes, and also hinder fibrosis by blocking the proliferation of fibroblasts [23]. In addition, oral rinse with topical corticosteroid can be used for vesiculo-erosive oral mucosal disease [24] and are effective for oral symptoms that occur in patients with chronic graft-versus-host disease who have undergone bone marrow transplantation [25]. They are also used to alleviate symptoms in patients with burning mouth syndrome [26]. Although there is no commercial dexamethasone gargle product available in Korea, dexamethasone gargles with concentrations of $0.01-0.06 \%$ are produced by mixing normal saline or distilled water with powder or liquid drugs and are used in otolaryngology, dentistry, and hematology-oncology (for burning mouth syndrome, oral lichen planus, or oral ulcers) [25]. In the present study, $0.05 \%$ dexamethasone solution was made by mixing 5 $\mathrm{mg} / 1 \mathrm{ml}$ of dexamethasone with $9 \mathrm{ml}$ of normal saline. Dexamethasone gargle is presumed to produce immediate effects (within minutes) directly on the upper respiratory tract, especially on the pharyngeal and laryngeal mucosa, similar to the effects of ketamine gargle [27]. In addition, endotracheal tube soaking with $0.05 \%$ dexamethasone solution can be performed for any patient.

According to the POST study by Bagchi et al. [13], intravenous injection of $0.2 \mathrm{mg} / \mathrm{kg}$ dexamethasone reduced the incidence of POST from $44 \%$ to $14 \%$ at 24 hours after surgery. Similarly, when the endotracheal tube cuff was soaked in dexamethasone, the incidence of POST fell from $63 \%$ to $26 \%$ during the initial 24 hours after surgery, signifying that topical use of dexamethasone is as effective on POST as intravenous injection of dexamethasone. Rudra et al. [15] examined the effects of ketamine gargles on POST, and found that the incidence of POST following a ketamine gargle was reduced from $60 \%$ to $25 \%$ at 24 hours after surgery. In the present study, the incidence of POST in patients who gargled with dexamethasone solution fell from $63 \%$ to $33 \%$ during the initial 24 hours after surgery, demonstrating a similar clinical outcome to those produced by ketamine gargles.

The pathology of POST involves irritation to the mucosa and cuff-induced pressure on the mucosa as well as a subsequent aseptic inflammatory process. In this context, the superior effects of dexamethasone gargle are presumed to be due to the fact that dexamethasone produces relatively stronger anti-inflammatory effects than ketamine by hindering the metabolism of arachidonic acid, inhibiting the release of cytokines (e.g., interleukin-2 and leukotriene-B4), and repressing the proliferation of fibroblasts.

Meanwhile, when Hung et al. [28] examined the effect on POST of spraying benzydamine hydrochloride on endotracheal tube cuffs, they found that the incidence of POST following spraying of the cuff with benzydamine hydrochloride dropped from $20 \%$ to $2 \%$ at 24 hours after surgery. In the present study, we performed endotracheal tube cuff soaking instead of spraying, but found similar results to those produced by benzydamine hydrochloride spray: the incidence of POST dropped from $63 \%$ to $26 \%$ during the initial 24 hours after surgery. Thus, we could predict that soaking endotracheal tube cuffs in dexamethasone solution also produced pharmacological actions through direct contact with the tracheal mucosa.

The current study had a few limitations. First, although topical steroid use is predicted to have fewer systemic adverse effects compared to intravenous injections, repeated and continuous use of steroids (even topical uses) can lead to adverse effects such as a Cushingoid appearance, hyperglycemia, suppression of the hypothalamic-pituitary-adrenal axis, hypertension due to sodium retention, potassium diuresis, oral candidiasis, psychosis, and peptic ulcers [29]. However, this study failed to take into consideration changes in blood concentrations induced by topical steroid applications and the subsequent possibility of systemic complications.

Second, this study only examined outcomes following laparoscopic cholecystectomy, which is usually a relatively short operation (completed within 45 minutes). As prior studies have reported high frequencies of POST associated with neck and gynecologic surgeries, and increasing frequencies of POST with increasing lengths of surgical procedures [6], further studies should be conducted to verify whether topical use of dexamethasone is also effective in these types of surgeries that are associated with high frequencies of POST. Third, sore throat is assessed on the basis of patients' subjective descriptions; in other words, the severity of pain may be expressed differently depending on a patient's experience and psychological state. There is a possibility that patients' answers during examinations may have been inaccurate, and it is difficult in practice to develop objective indices for sore throat.

In conclusion, gargling with $0.05 \%$ dexamethasone solution before intubation was effective in lowering the severity of POST, and soaking endotracheal tube cuffs in $0.05 \%$ dexamethasone solution before intubation was effective in lowering the incidence and severity of POST. 


\section{References}

1. Christensen AM, Willemoes-Larsen H, Lundby L, Jakobsen KB. Postoperative throat complaints after tracheal intubation. Br J Anaesth 1994; 73: 786-7.

2. Loeser EA, Bennett GM, Orr DL, Stanley TH. Reduction of postoperative sore throat with new endotracheal tube cuffs. Anesthesiology 1980; 52: 257-9.

3. Kang H, Kim KJ, Baek CW, Woo YC, Kim JY, Park SG. The effect of cuff pressure of laryngeal tube on postoperative sore throat after general anesthesia using N2O. Korean J Anesthesiol 2006; 50: 266-71.

4. Safavi M, Honarmand A, Fariborzifar A, Attari M. Intravenous dexamethasone versus ketamine gargle versus intravenous dexamethasone combined with ketamine gargle for evaluation of post-operative sore throat and hoarseness: A randomized, placebo-controlled, double blind clinical trial. Adv Biomed Res 2014; 3: 212.

5. Lee JH, Lee HK, Chun NH, So Y, Lim CY. The prophylactic effects of gabapentin on postoperative sore throat after thyroid surgery. Korean J Anesthesiol 2013; 64: 138-42.

6. Ahmed A, Abbasi S, Ghafoor HB, Ishaq M. Postoperative sore throat after elective surgical procedures. J Ayub Med Coll Abbottabad 2007; 19: $12-4$.

7. Jarahzadeh MH, Fuladgar B, Mirjalili MR, Rahimi-Bashar F, Dehghani MH, Barzegar K. Effect of local application of dexamethasone on reducing of post-surgical sore throat due to application of laryngeal mask airway. J Biol Today's World 2014; 3: 271-4.

8. Rashwan S, Abdelmawgoud A, Badawy AA. Effect of tramadol gargle on postoperative sore throat: A double blinded randomized placebo controlled study. Egypt J Anaesth 2014; 30: 235-9.

9. Banihashem N, Alijanpour E, Hasannasab B, Zarei A. Prophylactic effects of lidocaine or beclomethasone spray on post-operative sore throat and cough after orotracheal intubation. Iran J Otorhinolaryngol 2015; 27: 179-84.

10. Agarwal A, Gupta D, Yadav G, Goyal P, Singh PK, Singh U. An evaluation of the efficacy of licorice gargle for attenuating postoperative sore throat: a prospective, randomized, single-blind study. Anesth Analg 2009; 109: 77-81.

11. Haider HS, Al-Ali BT. The role of dexamethasone in reducing the severity of postoperative sore throat. Iraqi Postgrad Med J 2013; 12: 454-9.

12. Jeong SH, Park SH, Ok SM, Huh JY, Ko MY, Ahn YW. The effects of corticosteroid solutions in olp patients. J Oral Med Pain 2012; 37: 27-33.

13. Bagchi D, Mandal MC, Das S, Sahoo T, Basu SR, Sarkar S. Efficacy of intravenous dexamethasone to reduce incidence of postoperative sore throat: A prospective randomized controlled trial. J Anaesthesiol Clin Pharmacol 2012; 28: 477-80.

14. Park SY, Kim SH, Lee AR, Cho SH, Chae WS, Jin HC, et al. Prophylactic effect of dexamethasone in reducing postoperative sore throat. Korean J Anesthesiol 2010; 58: 15-9.

15. Rudra A, Ray S, Chatterjee S, Ahmed A, Ghosh S. Gargling with ketamine attenuates the postoperative sore throat. Indian J Anaesth 2009; 53: 40-3.

16. Saleem J, Athar S. Incidence of postoperative sore throat and cough: comparison of a polyvinylchloride tube and an armoured tube. Crit Care 2008; 12: 337.

17. Hwang GB, Lee SI, Park HS, Chung CJ. Incidence of sore throat after tracheal intubation and postoperative. Korean J Anesthesiol 1997; 33: 533-9.

18. Higgins PP, Chung F, Mezei G. Postoperative sore throat after ambulatory surgery. Br J Anaesth 2002; 88: 582-4.

19. Kim WJ, Oh HK. A clinical study of sore throat after endotracheal intubation. Korean J Anesthesiol 1977; 10: 41-5.

20. Huang YS, Hung NK, Lee MS, Kuo CP, Yu JC, Huang GS, et al. The effectiveness of benzydamine hydrochloride spraying on the endotracheal tube cuff or oral mucosa for postoperative sore throat. Anesth Analg 2010; 111: 887-91.

21. Sumathi PA, Shenoy T, Ambareesha M, Krishna HM. Controlled comparison between betamethasone gel and lidocaine jelly applied over tracheal tube to reduce postoperative sore throat, cough, and hoarseness of voice. Br J Anaesth 2008; 100: 215-8.

22. Rao M, Snigdha, Alai T, Vijay K. Instillation of $4 \%$ lidocaine versus air in the endotracheal tube (ETT) cuff to evaluate post intubation morbidity-a randomized double blind study. J Anesth Clin Sci 2013; 2: 19.

23. Streeten DH. Corticosteroid therapy. J AM Med Assoc 1975; 232: 944-8.

24. González-Moles MA, Scully C. Vesiculo-erosive oral mucosal disease--management with topical corticosteroids: (1) Fundamental principles and specific agents available. J Dent Res 2005; 84: 294-301.

25. Park AR, La HO, Cho BS, Kim SJ, Lee BK, Rhie JY, et al. Comparison of budesonide and dexamethasone for local treatment of oral chronic graft-versus-host disease. Am J Health Syst Pharm 2013; 70: 1383-91.

26. Choung YH, Cho MJ, Kim CH, Lee J, Kang SO. Preliminary results of steroid gargle treatment and clinical characteristics of patients with burning mouth syndrome. Korean J Otolaryngol-Head Neck Surg 2004; 47: 569-74.

27. Mayhood J, Cress K. Effectiveness of ketamine gargle in reducing the incidence of postoperative sore throat in patients undergoing airway instrumentation: A systematic review protocol. JBI Database System Rev Implement Rep 2014; 12: 82-8.

28. Hung NK, Wu CT, Chan SM, Lu CH, Huang YS, Yeh CC, et al. Effect on postoperative sore throat of spraying the endotracheal tube cuff with benzydamine hydrochloride, 10\% lidocaine, and 2\% lidocaine. Anesth Analg 2010; 111: 882-6.

29. González-Moles MA, Scully C. Vesiculo-erosive oral mucosal disease--management with topical corticosteroids: (2) Protocols, monitoring of effects and adverse reactions, and the future. J Dent Res 2005; 84: 302-8. 